Proceedings of the Edinburgh Mathematical Society (2011) 54, 309-319

DOI:10.1017/S0013091510000106

\title{
ON CONDITIONAL PERMUTABILITY AND SATURATED FORMATIONS
}

\author{
M. ARROYO-JORDÁ ${ }^{1}$, P. ARROYO-JORDÁ ${ }^{1}$ AND M. D. PÉREZ-RAMOS ${ }^{2}$ \\ ${ }^{1}$ Escuela Técnica Superior de Ingenieros Industriales, \\ Instituto Universitario de Matemática Pura y Aplicada, \\ Universidad Politécnica de Valencia, Camino de Vera $s / n$, \\ 46022Valencia, Spain (parroyo@mat.upv.es; marroyo@mat.upv.es) \\ ${ }^{2}$ Departament d'Àlgebra, Universitat de València, \\ Calle del Doctor Moliner 50, 46100 Burjassot (València), \\ Spain (dolores.perez@uv.es)
}

(Received 12 January 2010)

\begin{abstract}
Two subgroups $A$ and $B$ of a group $G$ are said to be totally completely conditionally permutable (tcc-permutable) in $G$ if $X$ permutes with $Y^{g}$ for some $g \in\langle X, Y\rangle$, for all $X \leqslant A$ and $Y \leqslant B$. We study the belonging of a finite product of tcc-permutable subgroups to a saturated formation of soluble groups containing all finite supersoluble groups.
\end{abstract}

Keywords: finite groups; products of subgroups; saturated formations; conditional permutability

2010 Mathematics subject classification: Primary 20D10; 20D40

\section{Introduction and results}

All groups considered in the paper are finite.

This paper is a contribution to the study of products of groups whose factors are linked by certain permutability properties. The origin of this research can be traced to Asaad and Shaalan [3], who provide criteria for the product of supersoluble groups to be supersoluble. Based on their paper, Maier $[\mathbf{2 2}]$ defines a group $G=H K$ to be the product of the totally permutable subgroups $H$ and $K$ if every subgroup of $H$ is permutable with every subgroup of $K$. We recall that a formation is a class $\mathcal{F}$ of groups closed under homomorphic images, such that $G / M \cap N \in \mathcal{F}$ whenever $G$ is a group and $M, N$ are normal subgroups of $G$ with $G / M \in \mathcal{F}$ and $G / N \in \mathcal{F}$. In this case the $\mathcal{F}$-residual $G^{\mathcal{F}}$ of $G$ is the smallest normal subgroup of $G$ such that $G / G^{\mathcal{F}} \in \mathcal{F}$. The formation $\mathcal{F}$ is saturated if $G \in \mathcal{F}$ whenever $G / \Phi(G) \in \mathcal{F}$, where $\Phi(G)$ denotes the Frattini subgroup of $G$. The following result was proved in $[\mathbf{4}, \mathbf{5}, \mathbf{7}]$. 
Theorem 1.1. Let $\mathcal{F}$ be a formation which contains the class $\mathcal{U}$ of all finite supersoluble groups. Let the group $G=G_{1} G_{2} \cdots G_{r}$ be a product of pairwise totally permutable subgroups $G_{1}, G_{2}, \ldots, G_{r}$. Then the following hold.

(i) If $G_{1}, G_{2}, \ldots, G_{r} \in \mathcal{F}$, then $G \in \mathcal{F}$.

(ii) Assume in addition that $\mathcal{F}$ is either saturated or a formation of soluble groups. If $G \in \mathcal{F}$, then $G_{1}, G_{2}, \ldots, G_{r} \in \mathcal{F}$.

Part (i) in this result was first proved for a saturated formation $\mathcal{F}$ such that $\mathcal{U} \subseteq \mathcal{F}$ in [22] (for $r=2$ ) and [9] (for $r$ arbitrary), generalizing a result in [3] for $\mathcal{F}=\mathcal{U}$, the saturated formation of all finite supersoluble groups.

Furthermore, products of totally permutable subgroups have been studied in depth both in the frameworks of formation theory $[\mathbf{4 - 1 0 , 2 2}]$ and in the theory of Fitting classes $[\mathbf{1 6}-\mathbf{1 8}]$. The structure of products of totally permutable subgroups is nowadays quite well understood. For instance, products $G=H K$ of totally permutable subgroups $H$ and $K$ are close to central products in the sense that the nilpotent residual $H^{\mathcal{N}}$ of $H$ centralizes $K$, and vice versa $[\mathbf{8}$, Theorem 1$] ; \mathcal{N}$ denotes the class of all finite nilpotent groups. Also $H \cap K \leqslant F(G)$, the Fitting subgroup of the group $G$ [22, Lemma 2], and $G$ modulo its $\mathcal{U}$-hypercentre $Z_{\mathcal{U}}(G)$ is a direct product of images of $H$ and $K$ [13, p. 859, Remarks (3)]. (The $\mathcal{U}$-hypercentre $Z_{\mathcal{U}}(G)$ of $G$ is the largest normal subgroup of $G$ such that every chief factor $X / Y$ of $G$ with $Y<X \leqslant Z_{\mathcal{U}}(G)$ is cyclic of prime order.)

More recently this development has been taken further by Guo et al. in [15], by considering a weaker condition of subgroup permutability, namely conditional permutability. We refer to $[\mathbf{1 4}, \mathbf{1 5}]$ and discuss the following concepts.

Definition 1.2. Let $G$ be a group. Two subgroups $X$ and $Y$ of $G$ are called conditionally permutable (c-permutable, for brevity) in $G$ if $X$ permutes with $Y^{g}$ for some element $g \in G$.

The subgroups $X$ and $Y$ are called completely conditionally permutable (cc-permutable) in $G$ if $X$ permutes with $Y^{g}$ for some element $g \in\langle X, Y\rangle$, the subgroup generated by $X$ and $Y$.

Two subgroups $A$ and $B$ of $G$ are said to be totally completely conditionally permutable (tcc-permutable) in $G$ if $X$ and $Y$ are cc-permutable in $G$ for all $X \leqslant A$ and $Y \leqslant B$.

Such a type of permutability conditions has been considered by other authors in extending classical results about the influence of permutability properties of certain families of subgroups on the structure of groups (see, for example, $[\mathbf{2}, \mathbf{1 4}, \mathbf{2 0}, \mathbf{2 3}]$ ).

This paper continues a study on products of subgroups, c-permutability and supersolubility carried out in [1]. Previous related results involving cc-permutability appear in $[\mathbf{1 5}, \mathbf{2 1}]$. In particular, the following result is obtained in [1, Theorem 2].

Theorem 1.3. Let the group $G=A B$ be the product of subgroups $A$ and $B$ such that every subgroup of $A$ is c-permutable in $G$ with every subgroup of $B$. Then $G^{\mathcal{U}}=A^{\mathcal{U}} B^{\mathcal{U}}$. In particular, if $A$ and $B$ are supersoluble, then $G$ is supersoluble. 
As mentioned in [1], in contrast to the previous research on products of totally permutable subgroups, this result does not extend to (saturated) formations containing $\mathcal{U}$, even in the universe of finite soluble groups (see Example 3.3). Nevertheless, we prove in this paper that positive results are possible if c-permutability is strengthened to cc-permutability. It is remarkable that c-permutability fails to satisfy the property of persistence in intermediate subgroups (see Example 3.4). Then cc-permutability appears when requiring c-permutability to satisfy this persistence property and becomes a stronger condition. However, significant structural properties of products of totally permutable subgroups, such as those mentioned previously, are missed when considering cc-permutability instead of permutability (see Examples 3.5 and 3.6).

The main aim of this paper is to prove the following result.

Theorem 1.4. Let $\mathcal{F}$ be a saturated formation of soluble groups containing $\mathcal{U}$. Let the group $G=G_{1} \cdots G_{r}$ be the product of pairwise permutable subgroups $G_{1}, \ldots, G_{r}$, for $r \geqslant 2$. Assume that $G_{i}$ and $G_{j}$ are tcc-permutable subgroups for all $i, j \in\{1, \ldots, r\}$, $i \neq j$. Then

(i) if $G_{i} \in \mathcal{F}$ for all $i=1, \ldots, r$, then $G \in \mathcal{F}$,

(ii) if $G \in \mathcal{F}$, then $G_{i} \in \mathcal{F}$ for all $i=1, \ldots, r$.

As a consequence, a stronger version of this theorem is obtained.

Corollary 1.5. Let $\mathcal{F}$ be a saturated formation of soluble groups containing $\mathcal{U}$. Let the group $G=G_{1} \cdots G_{r}$ be the product of pairwise permutable subgroups $G_{1}, \ldots, G_{r}$, for $r \geqslant 2$. Assume that $G_{i}$ and $G_{j}$ are tcc-permutable subgroups for all $i, j \in\{1, \ldots, r\}$, $i \neq j$. Then

(i) $G_{i}^{\mathcal{F}} \unlhd G$ for all $i=1, \ldots, r$,

(ii) $G^{\mathcal{F}}=G_{1}^{\mathcal{F}} \cdots G_{r}^{\mathcal{F}}$.

Analogous results to Corollary 1.5 for products of totally permutable subgroups and formations, either saturated or of soluble groups, containing $\mathcal{U}$ were obtained in $[\mathbf{4 , 7}]$ (for $r=2$ ) and [5] (for $r$ arbitrary). These results generalize a classical Doerk and Hawkes result which states that for a formation $\mathcal{F}$ of soluble groups the $\mathcal{F}$-residual respects the operation of forming direct products $[\mathbf{1 2}, \S \mathrm{IV}$, Theorem 1.18].

Section 2 is devoted to prove our main results. Some results and remarks about the behaviour of $\mathcal{F}$-projectors and $\mathcal{F}$-normalizers in such products of (soluble) groups, as well as the above-mentioned examples are collected in $\S 3$.

For notation and results on classes of groups we refer to [12]. In particular, $\sigma(G)$ denotes the set of all primes dividing the order of the group $G$. For subgroups $X, A$ of a group $G$, we define $\left\langle X^{A}\right\rangle=\left\langle x^{a}: x \in X, a \in A\right\rangle$; in particular, $\left\langle X^{G}\right\rangle$ is the normal closure of $X$ in $G$. For a prime $p$ the set of Sylow $p$-subgroups of $G$ is denoted by $\operatorname{Syl}_{p}(G)$. 


\section{Proof of the main results}

In order to prove Theorem 1.4 we need some previous results.

Lemma 2.1. Let the group $G=A B$ be the product of tcc-permutable subgroups $A$ and $B$. Then

(i) $X$ and $Y^{g}$ are tcc-permutable subgroups of $G$ for any $X \leqslant A, Y \leqslant B$ and $g \in G$,

(ii) for each $Y \leqslant B, A$ permutes with $Y^{b}$ for some $b \in B$.

Proof. (i) We take $g=b a \in G=A B=B A$ with $a \in A$ and $b \in B$. Then $Y^{g}=\left(Y^{b}\right)^{a}$ with $Y^{b} \leqslant B$.

We consider $X^{a^{-1}} \leqslant A$ and $Y^{b}=\left(Y^{g}\right)^{a^{-1}} \leqslant B$, which are tcc-permutable.

Let $X_{0} \leqslant X$ and $Y_{0} \leqslant Y^{g}$. Then $X_{0}^{a^{-1}} \leqslant X^{a^{-1}}$ and $Y_{0}^{a^{-1}} \leqslant Y^{b}$. Hence, there exists $t \in\left\langle X_{0}^{a^{-1}}, Y_{0}^{a^{-1}}\right\rangle$ such that $X_{0}^{a^{-1}}\left(Y_{0}^{a^{-1}}\right)^{t}=\left(Y_{0}^{a^{-1}}\right)^{t} X_{0}^{a^{-1}}$. Then $t^{a} \in\left\langle X_{0}, Y_{0}\right\rangle$ and

$$
\left(X_{0} Y_{0}^{t^{a}}\right)^{a^{-1}}=X_{0}^{a^{-1}}\left(Y_{0}^{t^{a}}\right)^{a^{-1}}=\left(Y_{0}^{t^{a}}\right)^{a^{-1}} X_{0}^{a^{-1}}=\left(Y_{0}^{t^{a}} X_{0}\right)^{a^{-1}}
$$

Therefore, $X_{0} Y_{0}^{t^{a}}=Y_{0}^{t^{a}} X_{0}$ with $t^{a} \in\left\langle X_{0}, Y_{0}\right\rangle$.

(ii) By hypothesis, $A$ permutes with $Y^{g}$ for some $g=b a \in\langle Y, A\rangle \leqslant B A, b \in B, a \in A$. Then $A=A^{a^{-1}}$ permutes with $Y^{b}=\left(Y^{g}\right)^{a^{-1}}$ and we are done.

Lemma 2.2 (Çunihin [11]). Let the group $G=H K$ be the product of subgroups $H$ and $K$. If $L \unlhd H$ and $L \leqslant K$, then $\left\langle L^{K}\right\rangle \unlhd G$; in particular, $L \leqslant \operatorname{Core}_{G}(K)$.

Proof. This follows easily since $L \leqslant\left\langle L^{G}\right\rangle=\left\langle L^{H K}\right\rangle=\left\langle L^{K}\right\rangle \leqslant \operatorname{Core}_{G}(K)$.

The following lemma is a key fact in our work. A corresponding result for products of totally permutable subgroups was proved in [22, Lemma 2(a)].

Lemma 2.3. Let the group $1 \neq G=A B$ be the product of tcc-permutable subgroups $A$ and $B$. Let $p$ be the largest prime divisor of $|G|$. Without loss of generality let $a \in A$ be a $p$-element of maximal order in $A \cup B$ and let $X_{0} \leqslant\langle a\rangle$ with $\left|X_{0}\right|=p$. Then

(i) $B^{g}$ normalizes $X_{0}$ for some $g \in G$,

(ii) $1 \neq\left\langle X_{0}^{A}\right\rangle \unlhd G$; in particular, $1 \neq X_{0} \leqslant \operatorname{Core}_{G}(A)$.

Proof. (i) Set $X=\langle a\rangle$. By hypothesis, $X B^{g}=B^{g} X$ for some $g \in G$. Arguing by induction on $|G|+|A|+|B|$ and using Lemma 2.1 (i), we may assume that $G=X B^{g}$ is the product of the tcc-permutable subgroups $X$ and $B^{g}$. Without loss of generality we may also set $B=B^{g}$.

Assume that $q$ is a prime divisor of $|B|$ such that $q \neq p$. By Lemma 2.1 (ii) and Sylow's theorems, $X$ permutes with $B_{q}$ for some $B_{q} \in \operatorname{Syl}_{q}(B)$. Hence, $X B_{q}$ is a product of the tcc-permutable supersoluble subgroups $X$ and $B_{q}$. It follows that $X B_{q}$ is supersoluble by Theorem 1.3 and, in particular, $B_{q}$ normalizes $X$. Hence, $B_{q}$ normalizes $X_{0}$. On the other hand, there exists $B_{p} \in \operatorname{Syl}_{p}(B)$ such that $X B_{p}=B_{p} X$ (if $p \notin \sigma(B)$, then $B_{p}=1$ ). 
If $X B_{p}<G$, we deduce by induction that $B_{p}^{u}$ normalizes $X_{0}$ for some $u \in X B_{p}$ which implies that $X_{0}$ is normal in $X B_{p}$. Consequently, $B=\left\langle B_{p}, B_{q} \mid q \neq p, q \in \sigma(B)\right\rangle$ normalizes $X_{0}$ and we are done.

Then, we may assume that $G=X B_{p}$ and $B=B_{p} \neq 1$.

We may assume that $X<G$ and consider a maximal subgroup $M$ of $G$ containing $X$. Then $M=X(B \cap M)$ and $X$ and $B \cap M$ are tcc-permutable subgroups of $M$. By induction we deduce that $(B \cap M)^{m}$ for some $m \in M$ normalizes $X_{0}$ and then $M$ normalizes $X_{0}$. Consequently, $M \leqslant N_{G}\left(X_{0}\right)=X N_{B}\left(X_{0}\right)$.

Assume that $N_{B}\left(X_{0}\right)<B$ and consider $b \in B \backslash N_{B}\left(X_{0}\right)$.

By hypothesis $X\langle b\rangle^{r}=\langle b\rangle^{r} X$ for some $r \in\langle X,\langle b\rangle\rangle$. Since $|X| \geqslant|\langle b\rangle|$, we can deduce from [19, $\oint$ VI, Satz 10.1] that $X_{0}$ is normal in $\langle b\rangle^{r} X$. We are now assuming that $G$ is a $p$-group and so $M \unlhd G$. Then $r=b^{i} s \in G=M\langle b\rangle$ for some $s \in M=N_{G}\left(X_{0}\right)$ and some integer $i$. Hence, $X_{0}^{b^{s}}=X_{0}^{b^{b^{i} s}}=X_{0}^{b^{r}}=X_{0}$ and so $X_{0}^{b}=X_{0}^{s^{-1} b}=X_{0}^{s^{-1}}=X_{0}$, which contradicts the choice of $b$ and concludes the proof of part (i).

(ii) From (i) we consider $B^{g}$, with $g \in G$, which normalizes $X_{0}$. Then $G=A B=$ $A\left(X_{0} B^{g}\right)$ and the result follows from Lemma 2.2 .

Remark 2.4. Lemma 2.3 states that for a group $1 \neq G=A B$ which is the product of tcc-permutable subgroups $A$ and $B$, there exists $1 \neq N \unlhd G$ such that either $N \leqslant A$ or $N \leqslant B$.

Lemma 2.5. Let the group $1 \neq G=G_{1} \cdots G_{r}$ be the product of pairwise permutable subgroups $G_{1}, \ldots, G_{r}$, for $r \geqslant 2$. Assume that $G_{i}$ and $G_{j}$ are tcc-permutable subgroups for all $i, j \in\{1, \ldots, r\}, i \neq j$. Then there exists $1 \neq N \unlhd G$ such that $N \leqslant G_{i}$ for some $i \in\{1, \ldots, r\}$.

Proof. Let $p$ be the largest prime divisor of $|G|$ and let $i \in\{1, \ldots, r\}$ such that the factor $G_{i}$ contains a $p$-element of $G_{1} \cup \cdots \cup G_{r}$ of maximal order. Then Lemma 2.3 implies that $G_{i}$ contains a non-trivial normal subgroup of $G$.

Lemma 2.6. Let the group $G=L N$ be the product of subgroups $L$ and $N$ with $N \unlhd G, N$ abelian and $N \cap L=1$. If $L$ is c-permutable in $G$ with a subgroup $X$ of $N$, then $L$ normalizes $X$. In particular, if $L$ is tcc-permutable with a subgroup $N_{0}$ of $N$, then $L$ normalizes every subgroup of $N_{0}$.

Proof. Assume that $L$ is c-permutable in $G$ with a subgroup $X$ of $N$. Then $L$ permutes with $X^{n}$ for some $n \in N$ as $G=L N$. Since $N$ is abelian, $L$ permutes with $X=X^{n}$ and then $X=(N \cap L) X=N \cap L X \unlhd L X$ and $L$ normalizes $X$. The rest is easily deduced.

Lemma 2.7. Let the group $G=A B$ be the product of tcc-permutable subgroups $A$ and $B$. Then $B$ normalizes $A^{\mathcal{U}}$, and vice versa.

Proof. Let $p$ be a prime divisor of $|B|$. By Sylow's theorems and Lemma 2.1 we deduce that $A$ permutes with $B_{p}$ for some $B_{p} \in \operatorname{Syl}_{p}(B)$, and, moreover, $A$ and $B_{p}$ 
are tcc-permutable subgroups. In particular, $A^{\mathcal{U}}=\left(A B_{p}\right)^{\mathcal{U}} \unlhd A B_{p}$ by Theorem 1.3. It follows now that $B$ normalizes $A^{\mathcal{U}}$.

Lemma 2.8. Let the group $1 \neq G=G_{1} \cdots G_{r}$ be the product of pairwise permutable subgroups $G_{1}, \ldots, G_{r}$, for $r \geqslant 2$. Assume that $G_{i}$ and $G_{j}$ are tcc-permutable subgroups for all $i, j \in\{1, \ldots, r\}, i \neq j$. Assume in addition that $G$ is a soluble primitive group; let $N$ be the unique minimal normal subgroup of $G$ and let $p$ be the prime divisor of $|N|$. Then either $G$ is supersoluble or the following conditions are satisfied:

(i) without loss of generality $N \leqslant G_{1}$;

(ii) $G_{2} \cdots G_{r}$ is a cyclic group whose order divides $p-1$;

(iii) there exists a maximal subgroup $M$ of $G$ with $\operatorname{Core}_{G}(M)=1$ such that $M=$ $\left(M \cap G_{1}\right)\left(G_{2} \cdots G_{r}\right)$ and $M \cap G_{1}$ centralizes $G_{2} \cdots G_{r}$.

Proof. Assume that $G$ is not supersoluble. Let $M$ be a maximal subgroup of $G$ with Core $_{G}(M)=1$. In particular, $G=N M, N \cap M=1$ and $N$ is an elementary abelian p-group. We prove the following.

Step $1\left(\boldsymbol{N} \not \subset \cap_{i=1}^{r} G_{i}\right)$. Otherwise $G_{i}=N\left(M \cap G_{i}\right)$ for each $i=1, \ldots, r$. It follows from Lemma 2.6 that $M \cap G_{i}$ normalizes every subgroup of $N$ for all $i=1, \ldots, r$. Hence, $N$ has order $p$ and $G$ is supersoluble: a contradiction.

By Lemma 2.5 and without loss of generality assume that $N \leqslant \cap_{i=1}^{s} G_{i}$ with $1 \leqslant s<r$ and $N \nless G_{j}$ for all $j=s+1, \ldots, r$. In particular, we note that $G_{i}=N\left(M \cap G_{i}\right)$ and $N \cap\left(M \cap G_{i}\right)=1$ for all $i=1, \ldots, s$.

Step $2\left(N \cap G_{j}=1\right.$ for all $\left.j=s+1, \ldots, r\right)$. Assume that $N \cap G_{j} \neq 1$ for some $j \in$ $\{s+1, \ldots, r\}$. By Lemma 2.6 we deduce that $N \cap G_{j}$ is normalized by $G_{i}$ for all $i=1, \ldots, s$. Then $N \cap G_{j} \unlhd G_{1} \cdots G_{s} G_{j}$ and Lemma 2.2 implies that $N \leqslant \operatorname{Core}_{G}\left(G_{s+1} \cdots G_{r}\right) \neq 1$. On the other hand, by Lemma 2.5 one of the factors $G_{s+1}, \ldots, G_{r}$ contains a minimal normal subgroup $R$ of $G_{s+1} \cdots G_{r}, R \neq N$. If $R \leqslant N$, it follows as above from Lemma 2.6 that $R$ is normal in $G$ and $R=N$ : a contradiction. Hence, $[R, N] \leqslant R \cap N=1$ and $R \leqslant C_{G}(N)=N$ : again a contradiction, which proves that $N \cap G_{j}=1$.

Step $3\left(G_{j}\right.$ normalizes every subgroup of $N$, and therefore $G_{j}$ is a cyclic group whose order divides $\boldsymbol{p}-\mathbf{1}$, for all $\boldsymbol{j}=s+\mathbf{1}, \ldots, \boldsymbol{r})$. Let $j \in\{s+1, \ldots, r\}$. It follows from Step 2 and Lemma 2.6 that $G_{j}$ normalizes every subgroup of $N$. Then for each $g \in G_{j}, n^{g}=n^{t}$ for some $t, 1 \leqslant t \leqslant p-1$, for every $n \in N$. Since $C_{G}(N)=N$ and $N \cap G_{j}=1$, we deduce that $G_{j}$ is a cyclic group whose order divides $p-1$.

Step $4(s=1)$. If $s \geqslant 2$ we deduce from Lemma 2.6 that $G_{i}$ normalizes every subgroup of $N$ for all $i=1, \ldots, s$. Consequently, $N$ has order $p$, by Step 3, and $G$ is supersoluble: a contradiction.

Step $5\left(G_{2} \cdots G_{r}\right.$ is a cyclic group whose order divides $p-1$ and $\left.\left[\boldsymbol{G}_{\boldsymbol{2}} \cdots \boldsymbol{G}_{\boldsymbol{r}}, \boldsymbol{G}\right] \leqslant \boldsymbol{N}\right)$. By Steps 3 and $4, G_{2} \cdots G_{r}$ normalizes every subgroup of $N$ and $G_{2} \cdots G_{r} \cap N=1$. The result is now deduced by using arguments as in Step 3; in particular we notice that $\left[G_{2} \cdots G_{r}, G\right] \leqslant C_{G}(N)=N$. 
Step 6 (without loss of generality we may assume that $G_{2} \cdots G_{r} \leqslant M$ and $M=\left(M \cap G_{1}\right)\left(G_{2} \cdots G_{r}\right)$; moreover, $M \cap G_{1}$ centralizes $\left.G_{2} \cdots G_{r}\right)$. By Step 5 and Hall's theorems, $G_{2} \cdots G_{r}$ is contained in a Hall $p^{\prime}$-subgroup of $G$ and then $G_{2} \cdots G_{r} \leqslant M^{g}$ for some $g \in G$. Without loss of generality we may set $M=M^{g}$. Since $G=G_{1}\left(G_{2} \cdots G_{r}\right)$, we have that $M=\left(M \cap G_{1}\right)\left(G_{2} \cdots G_{r}\right)$. Moreover, Step 5 implies also that $\left[G_{2} \cdots G_{r}, M \cap G_{1}\right] \leqslant M \cap N=1$.

We recall the following basic result on formations.

Lemma 2.9. Let $\mathcal{H}$ be a formation and let the group $G=H K$ be the product of subgroups $H$ and $K$ such that $H$ centralizes $K$. If $H, K \in \mathcal{H}$, then $G \in \mathcal{H}$.

Proof. Since $H$ centralizes $K, G$ is a factor group of the external direct product $H \times K$, and the result is easily deduced.

Proof of Theorem 1.4. (i) Assume that the result is not true and let the group $G=G_{1} \cdots G_{r}$ be a counterexample of minimal order. We notice that, for any normal subgroup $N$ of $G$, the factor group $G / N=\left(G_{1} N / N\right) \cdots\left(G_{r} N / N\right)$ satisfies the hypotheses of the theorem. Since $\mathcal{F}$ is a saturated formation, $G$ is a primitive group with a unique minimal normal subgroup, say $N$, and $G / N \in \mathcal{F}$; in particular, $G / N$ is a soluble group. By Lemma 2.5 we may assume without loss of generality that $N \leqslant G_{1} \in \mathcal{F}$. Hence, $N$ is soluble; consequently, $G$ is a soluble primitive group and Lemma 2.8 can be applied. We consider $M=\left(M \cap G_{1}\right)\left(G_{2} \cdots G_{r}\right)$ a maximal subgroup of $G$ as in Lemma 2.8. Let $F$ denote the canonical local definition of $\mathcal{F}$. Since $\mathcal{U} \subseteq \mathcal{F}$, we have that $G_{2} \cdots G_{r} \in F(p)$ for $p$ the prime divisor of $|N|$. Moreover, the fact that $G_{1} \in \mathcal{F}$ implies $M \cap G_{1} \in F(p)$. Therefore, $M=\left(M \cap G_{1}\right)\left(G_{2} \cdots G_{r}\right) \in F(p)$ by Lemma 2.9 as $F(p)$ is a formation. Since $G / C_{G}(N)=G / N \cong M \in F(p)$ and $G / N \in \mathcal{F}$, it follows that $G \in \mathcal{F}$ : a contradiction, which proves (i).

(ii) We argue as in (i) and consider $G=G_{1} \cdots G_{r}$ to be a counterexample of minimal order. We deduce here that $G$ has a unique minimal normal subgroup, say $N$, and again assume $N \leqslant G_{1}$ by Lemma 2.5. Moreover, now $G \in \mathcal{F}$ and so $G$ is soluble. Assume first that $G$ is primitive. From Lemma 2.8 and with the same notation we have that $G_{j} \in \mathcal{U} \subseteq \mathcal{F}$ for all $j=2, \ldots, r$. In addition, $M=\left(M \cap G_{1}\right)\left(G_{2} \cdots G_{r}\right) \cong G / N \in F(p)$ because $G \in \mathcal{F}$. Since $G_{2} \cdots G_{r}$ is a normal nilpotent subgroup of $M$, it follows from [12, $\S \mathrm{IV}$, Theorem 1.14] that $M \cap G_{1} \in F(p)$, which implies $G_{1}=N\left(M \cap G_{1}\right) \in \mathcal{F}$ : a contradiction.

Consider now the case $N \leqslant \Phi(G)$, the Frattini subgroup of $G$. We notice that $G_{i} N / N \in$ $\mathcal{F}$ for all $i=1, \ldots, r$. Assume that $N \leqslant G_{j}$ for some $j \neq 1$. Then for $k=1, j$, we have that $G_{k}=N F_{k}$, with $F_{k}$ an $\mathcal{F}$-projector of $G_{k}$. Since $N \leqslant G_{1} \cap G_{j}, N$ and $F_{k}$ are tcc-permutable subgroups and part (i) implies that $G_{k}=N F_{k} \in \mathcal{F}$. On the other hand, if $N \nless G_{j}$ for some $j \neq 1$, then $\operatorname{Core}_{G}\left(G_{j}\right)=1$ and we can deduce from Lemma 2.7 that $G_{j}^{\mathcal{U}}=1$, that is, $G_{j} \in \mathcal{U} \subseteq \mathcal{F}$. Consequently, it follows that $G_{1} \notin \mathcal{F}$ and $G_{j} \in \mathcal{U}$ for all $j=2, \ldots, r$. By the hypothesis, we notice that the $\mathcal{F}$-projector $F_{1}$ of $G_{1}$ permutes with $G_{j}^{n_{j}}$ for some $n_{j} \in N$ for each $j=2, \ldots, r$. Therefore, $F_{1}$ permutes with $\left\langle G_{2}^{n_{2}}, \ldots, G_{r}^{n_{r}}\right\rangle$ and $G=N F_{1}\left\langle G_{2}^{n_{2}}, \ldots, G_{r}^{n_{r}}\right\rangle=F_{1}\left\langle G_{2}^{n_{2}}, \ldots, G_{r}^{n_{r}}\right\rangle$, since $N \leqslant \Phi(G)$. Moreover, $F_{1}$ and 
$G_{j}^{n_{j}}$ for each $j=2, \ldots, r$, are tcc-permutable subgroups by Lemma 2.1 (i), which implies by Lemma 2.7 that $F_{1}^{\mathcal{U}}$ is normalized by $\left\langle G_{2}^{n_{2}}, \ldots, G_{r}^{n_{r}}\right\rangle$ and then $F_{1}^{\mathcal{U}}$ is normal in $G$. We notice that $G_{1}^{\mathcal{F}} \leqslant N$ is an abelian group, which implies by [12, $\S$ IV, Theorem 5.18] that $G_{1}^{\mathcal{F}} \cap F_{1}=1$. If $F_{1}^{\mathcal{U}} \neq 1$, then $G_{1}^{\mathcal{F}} \leqslant N \leqslant F_{1}^{\mathcal{U}} \leqslant F_{1}$ which implies $G_{1}^{\mathcal{F}}=1$, that is, $G_{1} \in \mathcal{F}$ : a contradiction. Therefore, $F_{1} \in \mathcal{U}$. Consequently, $G / N=N F_{1} G_{2} \cdots G_{r} / N \in \mathcal{U}$ by (i), which implies $G \in \mathcal{U}$ since $N \leqslant \Phi(G)$, and obviously $G_{i} \in \mathcal{U} \subseteq \mathcal{F}$ for all $i=1, \ldots, r$ : the final contradiction.

The obtained results (in particular, Theorem 1.4 and Lemmas 2.1 and 2.3) allow us to adapt the arguments in the proofs of [7, Lemmas 5 and 6] (see also the proof of Lemma 2.7) to deduce the following result.

Lemma 2.10. Let $\mathcal{F}$ be a saturated formation of soluble groups containing $\mathcal{U}$. Let the group $G=A B$ be the product of tcc-permutable subgroups $A$ and $B$. Then

(i) if $B \in \mathcal{F}$, then $G^{\mathcal{F}}=A^{\mathcal{F}}$,

(ii) $A^{\mathcal{F}}$ and $B^{\mathcal{F}}$ are normal subgroups of $G$.

Proof of Corollary 1.5. From Lemma 2.10 we deduce that $G_{i}^{\mathcal{F}}$ for all $i=1, \ldots, r$, and $K:=G_{1}^{\mathcal{F}} \cdots G_{r}^{\mathcal{F}}$ are normal subgroups of $G$. We notice that

$$
G / G^{\mathcal{F}}=\left(G_{1} G^{\mathcal{F}} / G^{\mathcal{F}}\right) \cdots\left(G_{r} G^{\mathcal{F}} / G^{\mathcal{F}}\right)
$$

satisfies the hypotheses of the result and then Theorem 1.4 (ii) implies that $K \leqslant G^{\mathcal{F}}$. By now considering $G / K=\left(G_{1} K / K\right) \cdots\left(G_{r} K / K\right)$, it follows that $G^{\mathcal{F}} \leqslant K$ from Theorem 1.4 (i). Consequently, $G^{\mathcal{F}}=K$.

\section{Some consequences and examples}

We deduce next some results about the behaviour of $\mathcal{F}$-projectors and $\mathcal{F}$-normalizers in products of tcc-permutable (soluble) subgroups. We follow here previous related results for products of totally permutable subgroups from $[\mathbf{4}, \mathbf{5}]$.

Corollary 3.1. Let $\mathcal{F}$ be a saturated formation of soluble groups containing $\mathcal{U}$. Let the group $G=A B$ be the product of tcc-permutable subgroups $A$ and $B$.

(i) There exist $\mathcal{F}$-projectors $X$ of $A$ and $Y$ of $B$ such that $X$ is permutable with $Y$. In this case $X Y$ is an $\mathcal{F}$-projector of $G$.

(ii) Assume in addition that $G$ is soluble. There exist $\mathcal{F}$-normalizers $U$ of $A$ and $V$ of $B$ such that $U$ is permutable with $V$ and $U V$ is an $\mathcal{F}$-normalizer of $G$.

Proof. (i) Let $X$ and $Y$ be $\mathcal{F}$-projectors of $A$ and $B$, respectively. Since $A$ and $B$ are tcc-permutable, there exists $g=b a \in G=B A$ with $a \in A$ and $b \in B$ such that $X$ permutes with $Y^{g}=Y^{b a}$. Then $X^{a^{-1}}$ permutes with $\left(Y^{g}\right)^{a^{-1}}=\left(Y^{b a}\right)^{a^{-1}}=Y^{b}$ and $X^{a^{-1}}$ is an $\mathcal{F}$-projector of $A$ and $Y^{b}$ is an $\mathcal{F}$-projector of $B$.

Assume now that $X$ and $Y$ are $\mathcal{F}$-projectors of $A$ and $B$ respectively, such that $X$ permutes with $Y$. By using the results in this paper, in particular, Lemmas 2.1 and 2.3 and Theorem 1.4, the proof of [4, Theorem B] can be adapted to deduce that $X Y$ is an $\mathcal{F}$-projector of $G$. 
(ii) We argue by induction on $|G|+|A|+|B|$. By using Theorem 1.4, Lemma 2.10 and the arguments in the proof of $[\mathbf{4}$, Theorem $\mathrm{C}]$, we may assume that $A \notin \mathcal{F}$ and deduce the following facts:

- there exists a normal subgroup $T$ of $A$ such that $T \leqslant A^{\mathcal{F}} \leqslant G^{\mathcal{F}}, T \leqslant F(A) \cap F(G)$;

- there exists a maximal subgroup $M$ of $A$ such that $A=T M=A^{\mathcal{F}} M=F(A) M$, whence $M$ is $\mathcal{F}$-critical in $A$.

By Lemma 2.1 (ii) there exists $a \in A$ such that $M^{a}$ permutes with $B$. Without loss of generality we may assume that $M$ permutes with $B$. If $M B$ is a proper subgroup of $G$, we notice that $G=T(M B)=G^{\mathcal{F}}(M B)=F(G)(M B)$ and $M B$ is a $\mathcal{F}$-critical maximal subgroup of $G$. In both cases, whether $G=M B$ or $M B<G$, the result follows, as in the proof of $[\mathbf{4}$, Theorem $\mathrm{C}]$, by induction and $[\mathbf{1 2}, \S \mathrm{V}$, Lemma 3.7], which states that $\mathcal{F}$-normalizers of $\mathcal{F}$-critical maximal subgroups of a group are $\mathcal{F}$-normalizers of the whole group.

Remark 3.2. Let $\mathcal{F}$ be a saturated formation of soluble groups containing $\mathcal{U}$. Let the group $G=G_{1} \cdots G_{r}$ be the product of the pairwise permutable subgroups $G_{1}, \ldots, G_{r}$, for $r \geqslant 2$. Assume that $G_{i}$ and $G_{j}$ are tcc-permutable subgroups for all $i, j \in\{1, \ldots, r\}$, $i \neq j$. It is an open question whether or not there exist $\mathcal{F}$-projectors $X_{i}$ of $G_{i}$ for each $i=1, \ldots, r$, such that $X_{1}, \ldots, X_{r}$ are pairwise permutable.

In this case $X_{1} \cdots X_{r}$ would be an $\mathcal{F}$-projector of $G$. The proof of this would follow as above, by using the results in this paper, in particular, Lemmas 2.1 and 2.5 and Theorem 1.4, and the proof of [5, Theorem 5] (see also the proof of [4, Theorem B]).

Assume in addition that the group $G=G_{1} \cdots G_{r}$ is soluble; equivalently, by Theorem 1.4 assume that the factors $G_{i}$ for all $i=1, \ldots, n$ are soluble. It is also an open question whether or not there exist $\mathcal{F}$-normalizers $X_{i}$ of $G_{i}$ for each $i=1, \ldots, r$, such that $X_{1} \cdots X_{r}$ is an $\mathcal{F}$-normalizer of $G$.

Next we gather the examples mentioned in $\S 1$. They are taken from $[\mathbf{1}]$ and included here for completeness.

Example 3.3 (Arroyo-Jordá et al. [1, Final Remark]). This example shows the failure of Theorem 1.4 when cc-permutability is weakened to c-permutability.

Let $G=\operatorname{Sym}(4)=A Y$ be the symmetric group of degree 4 , with $A=\operatorname{Alt}(4)$ the alternating subgroup of $G$ and $Y$ a subgroup of $G$ of order 2 generated by a transposition. Then every subgroup of $A$ is c-permutable in $G$ with every subgroup of $Y$; however, for $\mathcal{N}^{2}$ the saturated formation of metanilpotent groups, we have that $\mathcal{U} \subseteq \mathcal{N}^{2}, A, Y \in \mathcal{N}^{2}$ but $G \notin \mathcal{N}^{2}$.

Example 3.4 (Arroyo-Jordá et al. [1, Example 4]). We show now that c-permutability does not satisfy the property of persistence in intermediate subgroups, unlike cc-permutability.

We consider, as above, $G=\operatorname{Sym}(4)$ to be the symmetric group of degree 4 and $Y$ a subgroup of $G$ of order 2 generated by a transposition. Let $V$ be the normal subgroup of $G$ of order 4 and $X$ a subgroup of $V$ of order $2, X \neq Z(V Y)$. Then we observe that $X$ and $Y$ are c-permutable in $G$ but they are not c-permutable in $\langle Y, X\rangle$. 
The next examples show the failure of important structural properties of products of totally permutable subgroups when considering tcc-permutability.

Example 3.5 (Arroyo-Jordá et al. [1, Example 2]). We consider $G=\operatorname{Sym}(3)=$ $A B$, the symmetric group of degree 3 and the trivial factorization with $A=G$ and $B$ a Sylow 2-subgroup of $G$. Then $A$ and $B$ are tcc-permutable in $G$ but $B=B \cap A \nless F(G)$, the Fitting subgroup of $G$.

(We recall that if a group $G=H K$ is the product of totally permutable subgroups $H$ and $K$, then $H \cap K \leqslant F(G)[\mathbf{2 2}$, Lemma 2].)

Example 3.6 (Arroyo-Jordá et al. [1, Example 3]). Let $V=\langle a, b\rangle \cong Z_{5} \times Z_{5}$ and $Z_{6} \cong C=\langle\alpha, \beta\rangle \leqslant \operatorname{Aut}(V)$ as given by

$$
a^{\alpha}=a^{-1}, \quad b^{\alpha}=b^{-1} ; \quad a^{\beta}=b, \quad b^{\beta}=a^{-1} b^{-1} .
$$

Let $G=[V] C$ be the corresponding semidirect product of $V$ with $C$. Set $A=\langle\alpha\rangle$ and $B=V\langle\beta\rangle$. Then $G=A B$ and $A$ and $B$ are tcc-permutable subgroups of $G$ but they are not totally permutable. In fact, $B^{\mathcal{N}}=B^{\mathcal{U}}=V$ does not centralize $A$, in contrast to properties of products of totally permutable subgroups. We remark also that $Z_{\mathcal{U}}(G)=1$, and obviously $G$ modulo $Z_{\mathcal{U}}(G)$ is not a direct product of the images of $A$ and $B$.

(It is known that if $G=H K$ is the product of totally permutable subgroups $H$ and $K$, then $X^{\mathcal{N}}$ centralizes $Y$ for $\{H, K\}=\{X, Y\}$ [8, Theorem 1]; also $G$ modulo $Z_{\mathcal{U}}(G)$ is a direct product of the images of $H$ and $K$ [13, Remarks (3), p. 859].)

Acknowledgements. This research was supported by Proyectos MTM2007-68010C03-03 and MTM2010-19938-C03-02, Ministerio de Educación y Ciencia and FEDER, Spain.

\section{References}

1. M. Arroyo-Jordá, P. Arroyo-Jordá, A. Martínez-Pastor and M. D. PÉrezRAmos, On finite products of groups and supersolubility, J. Alg. 323 (2010), 2922-2934.

2. M. AsAad AND A. A. Heliel, On permutable subgroups of finite groups, Arch. Math. 80 (2003), 113-118.

3. M. Asaad And A. Shatlan, On the supersolvability of finite groups, Arch. Math. 53 (1989), 318-326.

4. A. Ballester-Bolinches, M. C. Pedraza-Aguilera and M. D. Pérez-Ramos, On finite products of totally permutable groups, Bull. Austral. Math. Soc. 53 (1996), 441-445.

5. A. Ballester-Bolinches, M. C. Pedraza-Aguilera and M. D. Pérez-Ramos, Finite groups which are products of pairwise totally permutable subgroups, Proc. Edinb. Math. Soc. 41 (1998), 567-572.

6. A. Ballester-Bolinches, M. C. Pedraza-Aguilera and M. D. Pérez-Ramos, Totally and mutually permutable products of finite groups, in Groups St Andrews 1997 in Bath I, London Mathematical Society Lecture Note Series, Volume 260, pp. 65-68 (Cambridge University Press, 1999).

7. A. Ballester-Bolinches and M. D. PÉrez-Ramos, A question of R. Maier concerning formations, J. Alg. 182 (1996), 738-747.

8. J. Beidleman And H. Heineken, Totally permutable torsion subgroups, J. Group Theory 2 (1999), 377-392. 
9. A. Carocca, A note on the product of $\mathcal{F}$-subgroups in a finite group, Proc. Edinb. Math. Soc. 39 (1996), 37-42.

10. A. Carocca And R. Maier, Theorems of Kegel-Wielandt type, in Groups St Andrews 1997 in Bath I, in London Mathematical Society Lecture Note Series, Volume 260, pp. 195201 (Cambridge University Press, 1999).

11. S. A. Çunihin, Simplicité de groupe fini et les ordres de ses classes d'éléments conjugués, C. R. Acad. Sci. Paris 191 (1930), 397-399.

12. K. Doerk And T. HaWkes, Finite soluble groups (Walter de Gruyter, Berlin, 1992).

13. M. P. GÁllego, P. Hauck And M. D. PÉRez-Ramos, On 2-generated subgroups and products of groups, J. Group Theory 11 (2008), 851-867.

14. W. Guo, K. P. Shum And A. N. SkibA, Conditionally permutable subgroups and supersolubility of finite groups, SE Asian Bull. Math. 29 (2005), 493-510.

15. W. Guo, K. P. Shum And A. N. Skiba, Criterions of supersolubility for products of supersoluble groups, Publ. Math. Debrecen 68 (2006), 433-449.

16. P. Hauck, A. Martínez-Pastor and M. D. PÉrez-Ramos, Fitting classes and products of totally permutable groups, J. Alg. 252 (2002), 114-126.

17. P. Hauck, A. Martínez-Pastor and M. D. PÉrez-Ramos, Products of pairwise totally permutable groups, Proc. Edinb. Math. Soc. 46 (2003), 147-157.

18. P. Hauck, A. Martínez-Pastor and M. D. PÉrez-Ramos, Injectors and radicals in products of totally permutable groups, Commun. Alg. 31 (2003), 6135-6147.

19. B. Huppert, Endliche Gruppen I (Springer, 1967).

20. H. Li AND G. QIAN, On PCM-subgroups of finite groups, JP J. Alg. Number Theory Applic. 12 (2008), 83-91.

21. X. Liu, W. Guo And K. P. Shum, Products of finite supersoluble groups, Alg. Colloq. 16 (2009), 333-340.

22. R. MAIER, A completeness property of certain formations, Bull. Lond. Math. Soc. 24 (1992), 540-544.

23. G. QIAN AND P. Zhu, Some sufficient conditions for supersolvability of groups, J. Nanjing Normal Univ. (Nature Sci.) 21 (1998), 15-21 (in Chinese). 
